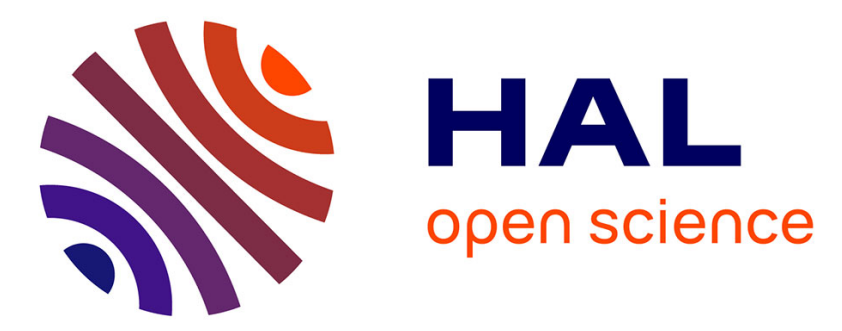

\title{
Validation of MFD-based models with microscopic simulations on real networks: Importance of production hysteresis and trip lengths estimation
}

\author{
Mahendra Paipuri, Ludovic Leclercq, Jean Krug
}

\section{To cite this version:}

Mahendra Paipuri, Ludovic Leclercq, Jean Krug. Validation of MFD-based models with microscopic simulations on real networks: Importance of production hysteresis and trip lengths estimation. Transportation Research Record, 2019, 2673 (5), pp478-492. 10.1177/0361198119839340 . hal-02381221

HAL Id: hal-02381221

https://hal.science/hal-02381221

Submitted on 26 Nov 2019

HAL is a multi-disciplinary open access archive for the deposit and dissemination of scientific research documents, whether they are published or not. The documents may come from teaching and research institutions in France or abroad, or from public or private research centers.
L'archive ouverte pluridisciplinaire HAL, est destinée au dépôt et à la diffusion de documents scientifiques de niveau recherche, publiés ou non, émanant des établissements d'enseignement et de recherche français ou étrangers, des laboratoires publics ou privés. 


\title{
Validation of MFD-based models with microscopic simulations on real networks: Importance of production hysteresis and trip lengths estimation
}

\author{
Mahendra Paipuri * \\ Univ. Lyon, ENTPE, IFSTTAR, LICIT \\ UMR_T 9401, F-69518, LYON, France \\ Tel: +33472047708 \\ Email: mahendra.paipuri@entpe.fr
}

9 Ludovic Leclercq

10 Univ. Lyon, ENTPE, IFSTTAR, LICIT

11 UMR_T 9401, F-69518, LYON, France

12 Tel: +33472047716

13 Email: ludovic.leclercq@entpe.fr

14 Jean Krug

15 Univ. Lyon, ENTPE, IFSTTAR, LICIT

16 UMR_T 9401, F-69518, LYON, France

17 Tel: +33472047064

18 Email: jean.krug@entpe.fr

$19 *$ Corresponding author

Paper submitted for presentation at the $98^{\text {th }}$ Annual Meeting Transportation Research Board, Washington D.C., January 2019 and for publication in Transportation Research Record: Journal of Transportation Research Board

Special call by AHB45 committee on "Advances in modeling and traffic management for large-scale urban network"

Word count: 7248 words +1 table $(\mathrm{s}) \times 250=7498$ words

February 17, 2019 
1 ABSTRACT

2 This work focuses on the application of accumulation-based and trip-based MFD approaches to 3 real transportation networks and discusses the calibration of the MFD shape and trip lengths es4 timation using a thorough validation of the network dynamics with micro-simulation data. This work not only investigates a classical unimodal approach to fit the production MFD, but also a bimodal MFD curve. Different methods of calibrating trip lengths in the reservoir are introduced to study the influence of trip lengths estimation on the accuracy of MFD models. MFD models are validated against micro-simulations that are carried out using the real OD matrix and demand that are estimated from the data of Lyon city in France. The proposed bimodal production MFD curve 10 captures the hysteresis in the production MFD to a good extent. Following, it is shown that the refined description of trip lengths gives more accurate estimates of accumulation evolution for the trip-based approach. Finally, a case is presented with a modified OD matrix to study the effect of OD matrix changes on accuracy of MFD simulations.

Keywords: Macroscopic Fundamental Diagram, production hysteresis, trip length estimation, accumulationbased model, trip-based model, micro-simulation, validation 


\section{INTRODUCTION}

There had been plenty of developments in employing Macroscopic Fundamental Diagrams (MFD) to predict the traffic state dynamics at the network level in the recent past. The MFD relates the density of vehicles to the mean flow in the network. This relationship was first introduced by Godfrey (1) and then reintroduced by Daganzo (2) to formulate new urban model. The existence of MFD under certain regularity assumptions is verified by Geroliminis and Daganzo (3). Since then, several applications like traffic state estimation (see e.g. Knoop and Hoogendoorn, Yildirimoglu and Geroliminis, 4, 5), perimeter control (see e.g. Keyvan-Ekbatani et al., Haddad and Mirkin, Ampountolas et al., 6, 7, 8), cruising-for-parking (see e.g. Cao and Menendez, Leclercq et al., 9, 10), etc. are proposed based on MFD approach.

Even though Geroliminis and Daganzo (3) reported a well-defined MFD for the city of Yokohama, it is to be noted that the empirical data from the traffic network of Yokohama approximately satisfies the regularity requirements proposed in Daganzo and Geroliminis (11). Some of them are homogeneous link distributions, slow varying demand, etc. Buisson and Ladier (12) first reported a bimodal MFD curve for the city of Toulouse using the empirical data of the traffic network. A clockwise hysteresis-like loop is observed, which is characterized by higher flow during loading and lower flow during unloading. Gayah and Daganzo (13) provided a deeper analytical investigation into the phenomenon of clockwise hysteresis and concluded that uneven congestion and drivers inability to re-route during the congestion peaks can be possible reasons for the hysteresis-like loops in MFD. Geroliminis and Sun (14) showed a similar hysteresis-like loop in MFD based on the empirical data of freeway networks. Their work attributed the cause of hysteresis phenomenon to the different degree of spatial heterogeneity in density during onset and offset of the congestion period. Ramezani et al. (15) proposed a parametrization model of production MFD (p-MFD) based on heterogeneity of link density in the network. Another factor that influences the shape of MFD is the demand pattern as shown in Leclercq et al. (16). Mahmassani et al. (17) showed that higher demand during congestion period results in the larger hysteresis loop in the MFD. Recently, Leclercq and Paipuri (18) proposed a deeper investigation of clockwise hysteresis phenomenon in the p-MFD by deriving the LWR solutions to an arterial case with internal bottleneck. They showed that when the network state is close to saturation, the congestion dynamics caused by bottlenecks with unequal shockwave speeds triggers the hysteresis shape in p-MFD. Following the empirical and analytical findings on production hysteresis in the literature, the importance of including hysteresis phenomenon in p-MFD for accurate description of network state dynamics is evident. Hence, first contribution of the present work is to include the hysteresis phenomenon in MFD-based simulations.

Another important question in formulating an accurate MFD simulator is definition of macroscopic trip lengths. Geroliminis and Daganzo (3) showed the existence of a linear relation between network production and trip completion rate and proposed the proportionality constant to be inverse of average trip length. However, Yildirimoglu and Geroliminis (5) compared the results of micro-simulation to the MFD-based simulations and concluded that using constant time invariant trip length to compute outflow has significant impact on the accuracy of the MFD-based simulation. Kouvelas et al. (19) also used constant trip length hypothesis in computing the outflow for their multi-reservoir simulations in the context of perimeter control. However, the authors stated that this assumption needs further investigation as strong fluctuations in demand and route choices can have an affect on outflow approximation. Leclercq et al. (16) showed that the internal trip patterns not only depend on the OD matrix, but also vehicle routing strategy inside the reser- 
voir. Therefore, the second contribution is to study the importance of level of description of trip lengths in a single reservoir setting in MFD-based simulation.

The accumulation-based MFD model is proposed by Daganzo (2) in the framework of single reservoir system. Later, this framework is extended to consider multiple trip lengths inside the reservoir in works of Geroliminis, Yildirimoglu et al. $(20,21)$. The main advantage of this model is being simple in terms of numerical resolution and computational complexity. Another MFD-based model, which gained significant attention in the recent past is the trip-based formulation. Originally based on idea proposed by Arnott (22), this approach is revisited by Leclercq et al., Daganzo and Lehe, Lamotte and Geroliminis (10, 23, 24). Mariotte et al. (25) refined this idea to propose the so-called event-based model for a single reservoir system in the framework of trip-based MFD models. The main assumption of this approach is that all the vehicles travel at the same mean speed given by the MFD at a given time and exit the reservoir after they finish their individually assigned trip lengths. This model is computationally more demanding compared to its counterpart. However, trip-based model addresses few limitations of accumulation-based model which can be found in Mariotte et al. (25). More recently, Mariotte and Leclercq (26) extended the trip-based framework to multiple reservoirs systems that can have multiple trip lengths in each reservoir. Their work proposed a novel way to model the congestion spill-backs in the trip-based formulation. However, Leclercq and Paipuri (18) showed that no model is perfect and the tripbased exhibits inconsistent outflow patterns close to saturation. This can be avoided by monitoring the outflow, however the travel times in the reservoir are no longer consistent with trip lengths and mean speed.

There have been complex formulations proposed in the MFD-based simulation approaches in the literature. The inclusion the production hysteresis and definition the trip lengths inside the reservoir of MFD models are still ongoing research questions. Most of the MFD-based simulation approaches are applied to idealized networks and there are only very few detailed validations on real networks. Hence, the contribution of this study is two-fold namely, a detailed investigation into MFD calibration and trip length estimation and a thorough validation of the MFD-based simulations on real network of $6^{\text {th }}$ district of Lyon city (Lyon 6), France. A conventional unimodal MFD and a bimodal MFD with hysteresis patterns are computed from micro-simulation data. This work is the first to consider the clockwise hysteresis-like loop in the p-MFD for both accumulationbased and trip-based models. Another contribution of this work is to establish the importance of the level of description of trip length distributions in reservoir simulation. The individual trip lengths are known a priori from the micro-simulation data and therefore, an accurate model can be built by considering each individual trip length in the MFD simulators. Apart from the individual trip lengths, other definitions like single mean trip, trip based on OD, etc. are considered in the present work. The accuracy of different approaches of trip lengths are presented. The given OD matrix is modified artificially to study the sensitivity of MFD-based simulations on the changes in OD pattern.

The paper is organized as follows: section 2 presents the Lyon 6 network description, section 3 discusses the calibration of p-MFD and trip length estimation methods, section 4 briefs about MFD simulator's accuracy and finally section 5 presents the numerical results. 


\section{LYON 6 NETWORK DESCRIPTION} Network characteristics

Figures 1a and 1b show the map of Lyon 6 area and the link level description of the network, respectively. The district covers a total area of $3.77 \mathrm{~km}^{2}$. The area analyzed in micro-simulation comprises of Lyon 6, part of Lyon 3 and Villeurbanne (Lyon 63V) area, France as shown in Fig. 1c. The whole network is segregated into 75 origins and destinations of which 21 zones belong to Lyon 6 area. This simulation set-up consists of transfer trips that start and finish outside Lyon 6 area by transversing through Lyon 6 and more importantly, internal trips that start and finish inside Lyon 6 network. Public transport, i.e., buses are also considered in the simulation setup. Hence, the total outflow corresponds to the sum of trip completion rate of internal trips including buses and flow of vehicles that cross border of Lyon 6 area.

Three different scenarios, a free-flow case where peak demand is below the network saturation, a congestion case with peak demand close to network saturation and a congestion case with modified OD matrix, are considered in the present work for the morning peak hour case from $06 h 30$ to $13 h 30$. Figure 2a presents the demand that is estimated from the loop-detectors data that is normalized by the total demand over $24 h r$ for the three different scenarios. The free-flow demand (in blue) is used for free flow scenario, whereas the network saturation case (in red) is used for congestion scenario with original and modified OD matrices. Figure $2 b$ shows the respective actual demand from all different trips aforementioned inside the Lyon 6 network for two different demand levels. Time-dependent OD matrix is estimated from the empirical data of the Lyon city network. The estimated demand is only applicable to cars and there is no reliable data available for trucks. Hence, the truck demand is assumed to be $5 \%$ of the car demand in the present simulations. Based on the OD matrix and route definitions, there are 19080 different trips using the original OD matrix and network saturation demand pattern inside the Lyon 6 network and their corresponding distribution is shown in Fig. $2 \mathrm{c}$ and mean trip length is $1505 \mathrm{~m}$. For the case of modified OD matrix, the trip length distribution is presented in Fig. 2d with mean trip length of $1652 \mathrm{~m}$.

\section{Micro-simulation settings}

A triangular fundamental diagram is assumed with identical parameters for each vehicle category. Two classes of vehicles are considered, namely cars and trucks. The parameters for cars used are: free-flow speed, $u=25 \mathrm{~m} / \mathrm{s}$, wave speed, $w=5.88 \mathrm{~m} / \mathrm{s}$ and jam density, $\kappa=0.17 \mathrm{veh} / \mathrm{m}$, where as for trucks: free-flow speed, $u=22 \mathrm{~m} / \mathrm{s}, w=5.88 \mathrm{~m} / \mathrm{s}$ and $\kappa=0.075 \mathrm{veh} / \mathrm{m}$. It is to be noted that the $u$ is maximum free-flow speed and all vehicles will adjust the free-flow speed to the link speed limits, which are given by the network data. The traffic signal settings at the intersections are implemented based on the real data. The micro-simulations are computed using Symuvia platform that is developed within the research laboratory. The platform is based on the Newell's car following law (see e.g. Newell, Leclercq et al., 27, 28). A static traffic assignment based on Logit's model (see e.g. Dial, 29) is used for all OD pairs. The duration of the simulation is $7 \mathrm{hr}$ in all the results presented.

\section{CALIBRATION OF PRODUCTION MFD AND TRIP LENGTHS ESTIMATION Influence of aggregation period}

Firstly, a preliminary study is made to understand the influence of aggregation period in the calibration of p-MFD. A reference scenario with a peak demand close to network saturation is considered. Different aggregation periods of $\{180,360,420,600,720\} \mathrm{sec}$ are considered. Some of the 


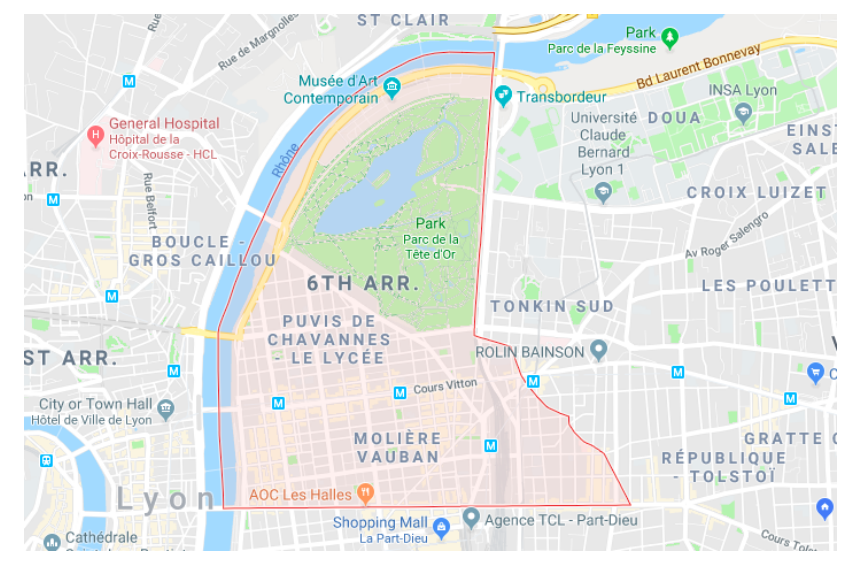

(a) Map of the Lyon 6 @Google Maps 2018.

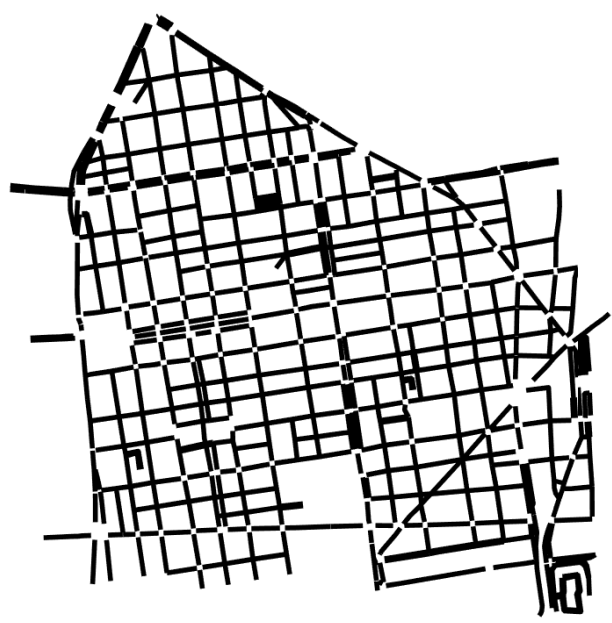

(b) Link level representation of the Lyon 6.

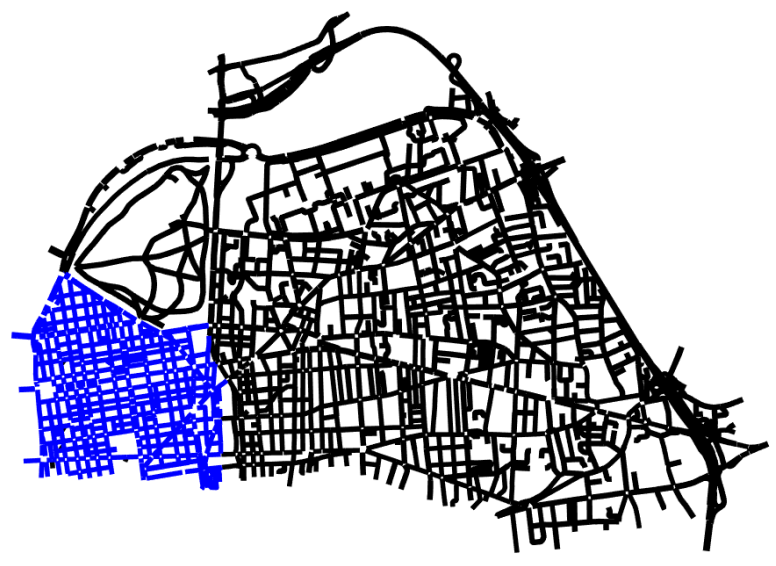

(c) Link level representation of Lyon 6, Lyon 3 and Villeurbanne networks. Lyon 6 is highlighed in blue.

FIGURE 1 : Lyon 6 network: map of the area, its link level description and whole network considered in micro-simulation. 


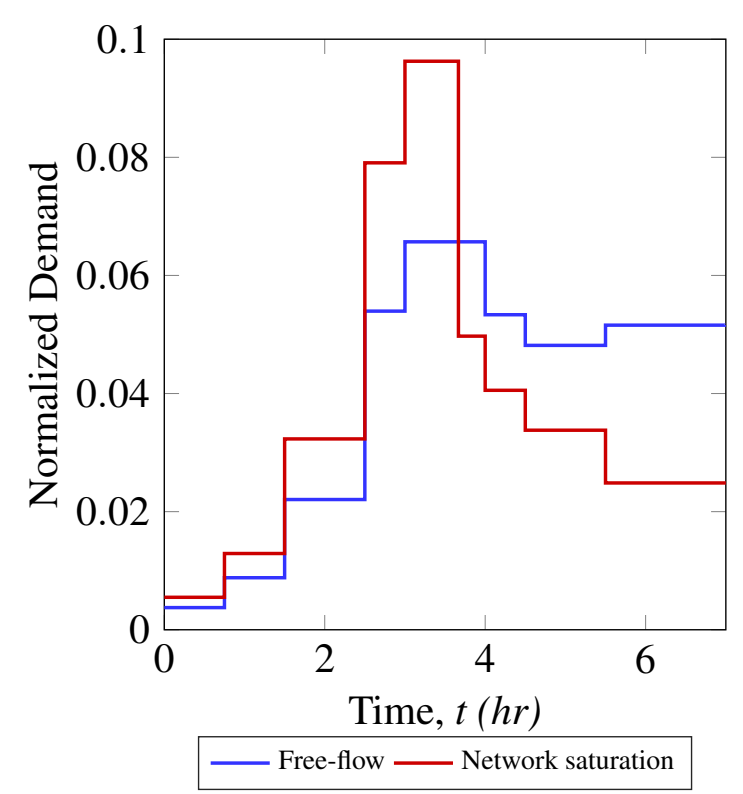

(a) Normalised demand for considered scenarios.

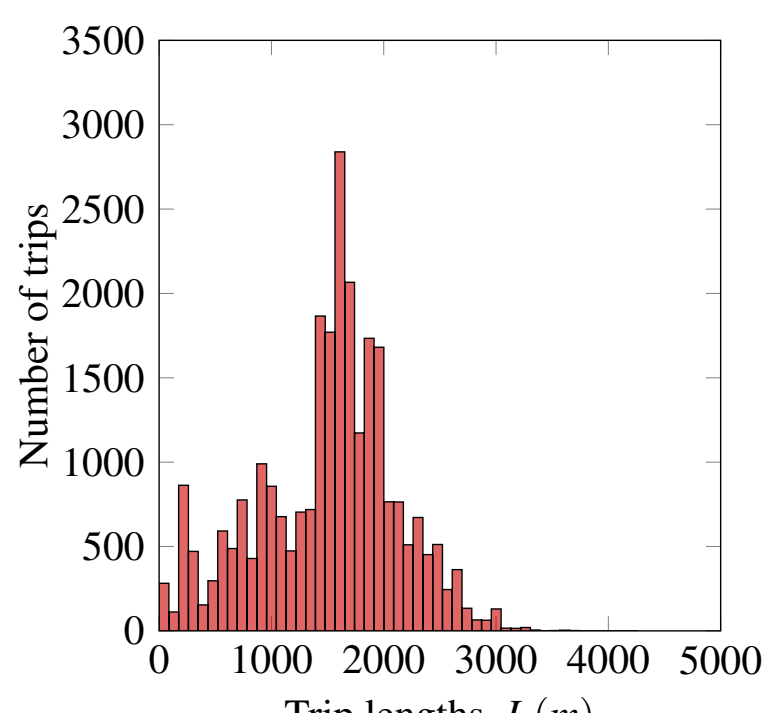

(c) Trip lengths distribution in Lyon 6 network for original(d) Trip lengths distribution in Lyon 6 network for modified OD matrix with network saturation scenario.

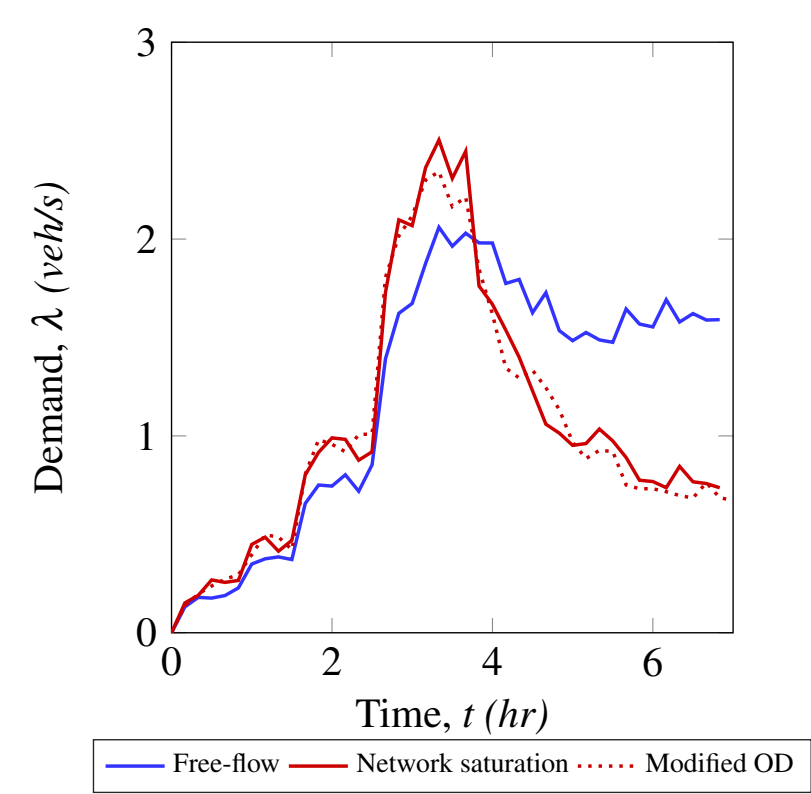

(b) Actual demand in veh/s for considered scenarios in Lyon 6 network.

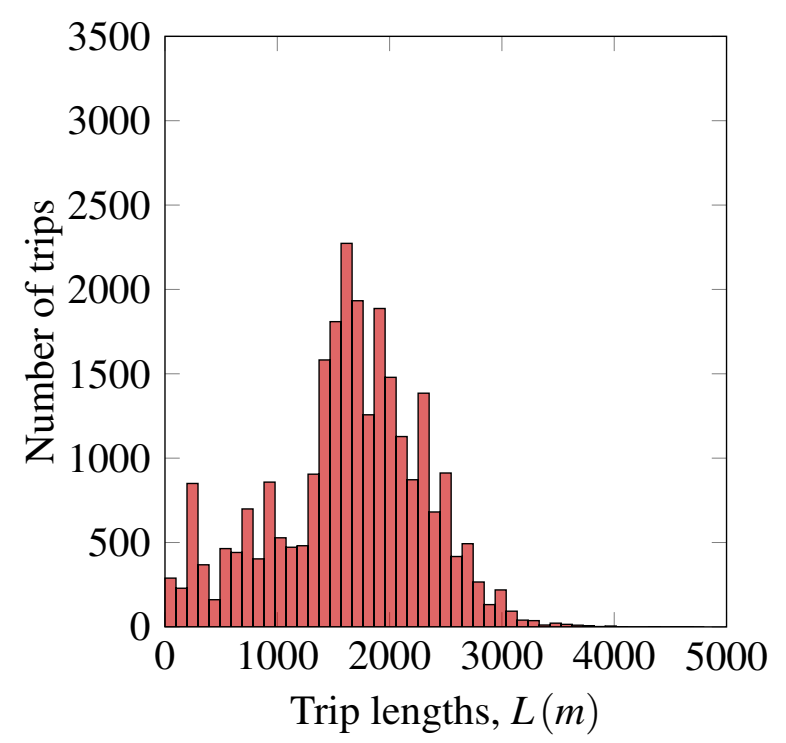

FIGURE 2 : Lyon 6 network: map of the area, its link level description, demand for different cases and trip lengths distribution. 

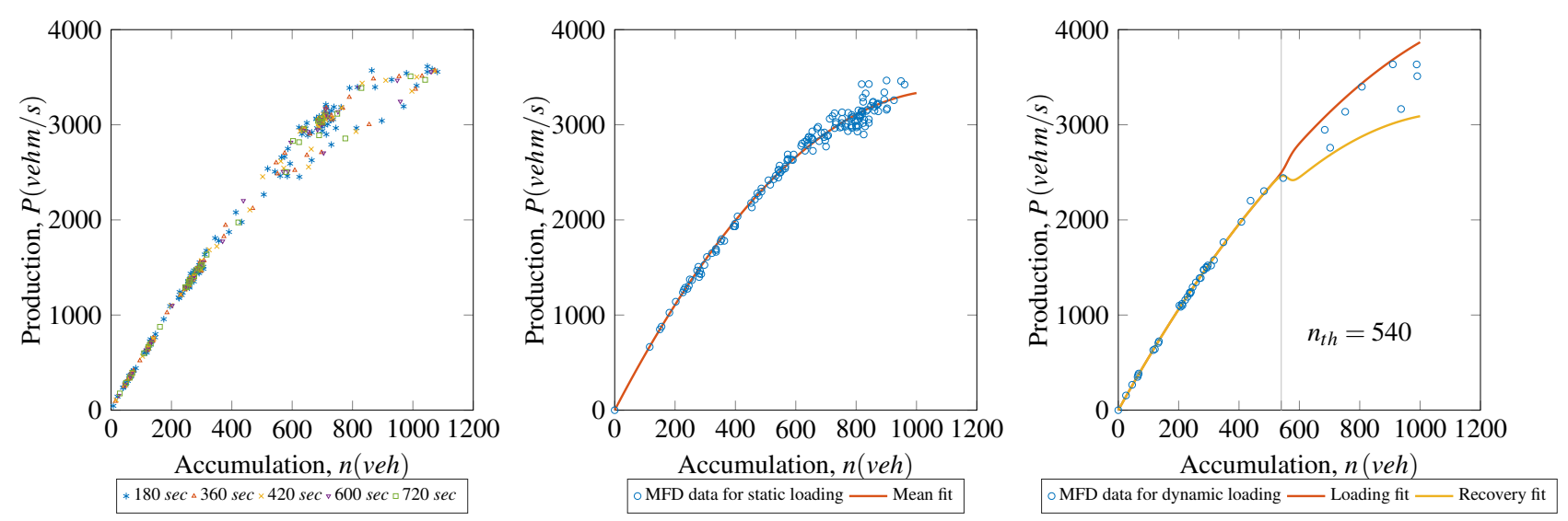

(a) p-MFD data with different aggrega-(b) p-MFD data with static loading and(c) p-MFD data with dynamic loading tion periods. its unimodal fit.

and its bimodal fit.

FIGURE 3 : Lyon 6 network: influence of aggregation period, calibration of p-MFD with static and dynamic loadings and their corresponding unimodal and bimodal MFD fits.

signal cycle settings in the network are in the order of $100 \mathrm{sec}$ and hence, an aggregation period of less than $100 \mathrm{sec}$ would not be consistent with MFD settings and would result in high scatter profile. The microscopic variables Total Traveled Time (TTT) and Total Traveled Distance (TTD) are aggregated over the considered periods and the corresponding vehicle accumulation $(n)$ and production $(P)$ are computed. Figure 3a presents the p-MFD with different aggregation periods considered. It can be noticed that the MFD is well-captured and it is quite independent of the aggregation period. Therefore, in the present work an aggregation period of $600 \mathrm{sec}$ is used in all computations.

\section{Unimodal and bimodal MFD fits}

Production MFD data is first calibrated by loading the considered network with different levels of static demand until a steady state is obtained in the micro-simulations. Figure $3 b$ presents the data points on accumulation-production plane obtained for different demand levels. The network loading in the free-flow regime results in a good steady state approximation where changes in both production and accumulation are negligible with time. However, close to the network saturation scatter in the MFD data can be noticed, which is the consequence of pseudo steady states. A conventional unimodal fit is computed for the steady state MFD data which relates the mean accumulation with mean production in Fig. 3b. Note that the unimodal MFD fit cannot account for the scatter of MFD data close to network saturation. This computed unimodal fit can be expressed as follows,

$$
P_{u m}(n)=-0.0024 n^{2}+5.9160 n,
$$

where $P_{u m}(n)$ is unimodal fit of the p-MFD.

Besides the conventional unimodal fit, this work proposes the bimodal MFD fit to distinguish between network loading and recovery phases. To accomplish the task, a micro-simulation with dynamic demand corresponding to network saturation shown in Fig. $2 b$ is carried out and the corresponding p-MFD data is plotted in Fig. $3 \mathrm{c}$ in blue circles. It can be observed that the values of production in network loading and recovery are different owing the phenomenon of hysteresis. 
Hence, in the present work a bimodal MFD is computed using the hysteresis loop of the dynamic simulation and as stated earlier, unimodal p-MFD fit is estimated from static demand loadings.

It is noticed that until $n \leq 540 v e h$, the scatter of the MFD data in dynamic loading case is very low and hence, it is possible to represent this data by a unique parabolic fit. For $n>540$ veh, two parabolic curves are fitted that follow the loading and recovering MFD points as shown in Fig. 3c. The relation between production and accumulation can be expressed as follows,

$$
P_{b m}(n)= \begin{cases}-0.0021 n^{2}+5.72 n & n \leq 540 \\ -0.0020 n^{2}+5.89 n & n>540 \& \frac{\Delta n}{n} \geq 0 \quad \text { (Loading) } \\ -0.0025 n^{2}+5.55 n & n>540 \& \frac{\Delta n}{n}<0 \quad \text { (Recovery) }\end{cases}
$$

In order to avoid the discontinuity at $n=540$, the curves are joined using a smoothening function. In the present work, the trigonometric function $\tanh x$ is used to join the curves. Therefore, during the loading of the network, loading fit in Fig. 3c is used to maximize the network performance. Similarly, during unloading phase, recovery fit is used to reproduce the hysteresis phenomenon observed in micro-simulations. The critical accumulation, $n_{c}$, and the corresponding critical production, $P_{c}$, are $900 \mathrm{veh}$ and $3680 \mathrm{vehm} / \mathrm{s}$, respectively. Using the data points on the congestion part of the MFD, jam accumulation, $n_{j}$, is extrapolated to $3300 \mathrm{veh}$. In the implementation, $\Delta n(t)=n(t)-n(t-60)$, where $t$ is time in seconds and a tolerance is used for $\frac{\Delta n(t)}{n(t)}$ to avoid local oscillations.

\section{Trip lengths estimation}

The total number of trip lengths vary depending on the demand pattern and OD matrix. There are a total of 22226 trips in the Lyon 6 network corresponding to the free-flow demand pattern shown in Figs. 2a and 2b. As stated earlier, there are total of 19080 and 26120 trips for the case of network saturation scenario with original and modified OD matrices, respectively. The reason for having more trips in free-flow scenario than the network saturation with original OD matrix is that demand is kept at nominal level after the peak in the case of free-flow, while demand is reduced to a low value in the case of network saturation as shown in Fig. 2 b.

In order to demonstrate the importance of level of description of trip lengths, four different methods of trip length estimation is proposed in this work.

Mean trip: Only one trip length value is considered inside the reservoir for all trips. It is defined as arithmetic mean of all trip lengths. Hence, the mean trip length depends on the scenario under consideration.

OD trips: Depending on the origin and destination of each trip with respect to Lyon 6 area, four different types of trips can be identified: Trips starting outside and ending outside, Trips starting inside and ending inside, Trips starting outside and ending inside and Trips starting inside and ending outside. The mean trip length per trip type is computed and given as length to the respective trip.

Similar trips: Several trips are clustered into bins based on the range of trip lengths. The mean trip length inside each bin is given as trip length to the corresponding trip. 
Individual trips: Each individual trip is considered and the corresponding trip length is assigned to each trip in the MFD simulations.

Hence, from the aforementioned definition of trip length estimations methods, it can be observed that the level of description of trip lengths increases from single mean trip to individual trips. In other words, trip lengths are exact in case of individual trips and least accurate in case of single mean trip. In the numerical results, different methods of trip length estimation are compared to demonstrate the influence of trip lengths description on accuracy of traffic state dynamics in MFD approaches.

\section{MFD-BASED SIMULATION TECHNIQUES}

\section{Accumulation-based model}

The following expression governs the dynamics in a single reservoir context with multiple trip lengths based on the conservation equation (see e.g. Daganzo, 2),

$$
\frac{d n_{i}}{d t}=q_{\text {in }, i}(t)-q_{\text {out }, i}(t) \text { for } i=1, \ldots, \text { ntrips },
$$

where $n_{i}$ is the partial vehicle accumulation for the trip $i, q_{i n, i}$ and $q_{\text {out }, i}$ are the inflow and outflow, respectively. The computation of effective inflow and outflow is discussed in-detail in $(26,30)$. The outflow of the accumulation model is governed by outflow demand function, $O_{i}\left(n_{i}, n\right)$, which is defined as,

$$
O_{i}\left(n_{i}, n\right)= \begin{cases}\frac{n_{i}}{n} \frac{P(n)}{L_{i}} & n<n_{c} \\ \frac{n_{i}}{n} \frac{P_{c}}{L_{i}} & n \geq n_{c},\end{cases}
$$

where $n$ is total accumulation on all trips, i.e., $\sum_{i=1}^{n t r i p s} n_{i}, L_{i}$ is the trip length of trip $i$ and $P(n)$ is the production computed from MFD. In accumulation-based model, outflow or trip-completion rate, $G(n)$, is approximated as $\frac{P(n)}{L}$ and hence, it is also referred as PL (production over trip length) model.

Hence, the conservation equation (3) can be rewritten using eq (4) as follows,

$$
\frac{d n_{i}}{d t}=q_{i n, i}(t)-O_{i}\left(n_{i}, n\right) \text { for } i=1, \ldots, n t r i p s .
$$

The Ordinary Differential Equation (ODE) in eq (5) is numerically resolved using first-order explicit Euler method as follows,

$$
\frac{n_{i}^{t+\Delta t}-n_{i}^{t}}{\Delta t}=\lambda_{i}^{t}-O\left(n_{i}^{t}, n^{t}\right)
$$

where $\lambda_{i}^{t}$ is the demand and $\Delta t$ is the time step. In the present work, a time step of $1 \mathrm{sec}$ is used in all computations. Depending on the demand level for a given route, there can be as few as 1 veh per trip during whole simulation time. Considering each individual trip in accumulation-based model can add significant numerical diffusion into the scheme. Hence, the case of individual trips is not considered for accumulation-based model. 


\section{Trip-based model}

The trip-based approach (see e.g. Arnott, 22) is based on the principle that all vehicles travel at the same speed at any given time. The vehicles leave the reservoir once they finish their assigned trip length. If a vehicle entered at time $t$ traveled a distance $L$ in time $T(t)$, the trip-based model can be mathematically expressed as,

$$
L=\int_{t-T(t)}^{t} V(n(s)) d s .
$$

The mean speed $V(n)$ is computed from p-MFD, i.e., $V(n)=P(n) / n$. In the present work, eventbased resolution proposed in Mariotte et al., Lamotte and Geroliminis $(25,31)$ is used in the trip-based formulation. In the event-based formulation, the entry and the exit of each vehicle is considered as an event and network variables like accumulation, mean speed etc., are updated for each event. As mentioned earlier, entry times of each vehicle is known a priori from microsimulation and hence, it is an input to event-based formulation. Each vehicle travels with mean speed that evolves based on traffic dynamics. Once the vehicle finishes its assigned trip length, the considered trip is completed and vehicle is removed from the reservoir. As proposed in Leclercq and Paipuri, Mariotte and Leclercq $(18,26)$, the outflow (or trip completion rate) is bounded by the maximum capacity and to sustain the outflow to maximum capacity when network reaches saturation to avoid causality effect (see e.g. Merchant and Nemhauser, Friesz et al., 32, 33). The maximum capacity of the reservoir is computed from the micro-simulation results. Even though different trip lengths are considered, a single queue of vehicles is monitored during the simulation and maximum outflow limitation is applied to the single queue. This avoids the need of defining the maximum outflow for each trip length defined. Owing to the formulation of event-based scheme, it is possible to take all the different trip lengths into account while computing the traffic dynamics. Hence, along with other cases of trip lengths described earlier, individual trip lengths are also considered for event-based formulation.

\section{VALIDATION RESULTS FOR THE REFERENCE SCENARIOS}

\section{Free flow traffic state scenario}

Firstly, a free flow scenario is considered where the peak demand is less than that of the network saturation state. Figure 4 shows the different state variables like accumulation, mean speed, outflow, etc. The normalized demand curve shown in Fig. 2a (in blue) is given to each OD matrix in micro-simulation. Since, the flow between an OD pair that transverse through Lyon 6 with origin/destination outside Lyon 6 cannot be predicted a priori, demand is computed from microsimulation data rather than the actual OD matrix data. In the case of trip-based approach, the starting times of each trip is the input and therefore, the micro-simulation data can be used directly. However, in the case of accumulation-based approach, the demand per each trip is computed by taking the first derivative of cumulative curve of entering vehicles per trip. Since the exact trip starting times are known a priori from the micro-simulation data, entry supply function is not considered in the present work. This is done to avoid the discrepancies from the entry flow function, as the primary objective of the work is to study the accuracy of models with respect to p-MFD and trip lengths calibration. However, in the context of multi-reservoir settings, entry supply function must be defined and it is out of the scope of present work.

Figure 4a shows the evolution of accumulation with time for both MFD simulators with unimodal p-MFD fit along with the comparison to micro-simulation data. Bimodal p-MFD fit is not considered in this case as the considered demand peak is not high enough to produce hysteresis 


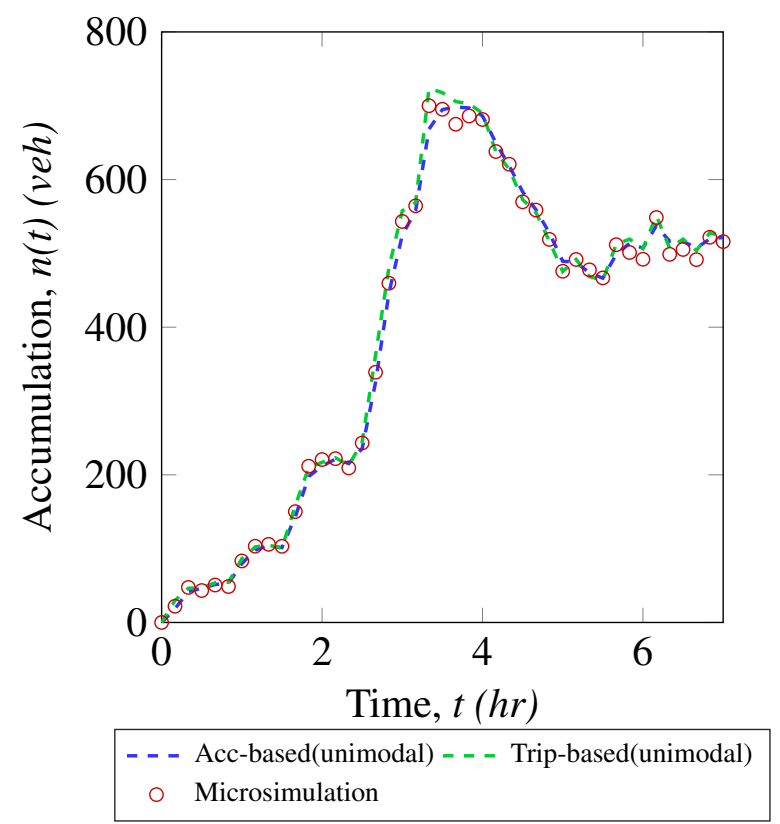

(a) Evolution of accumulation with time.

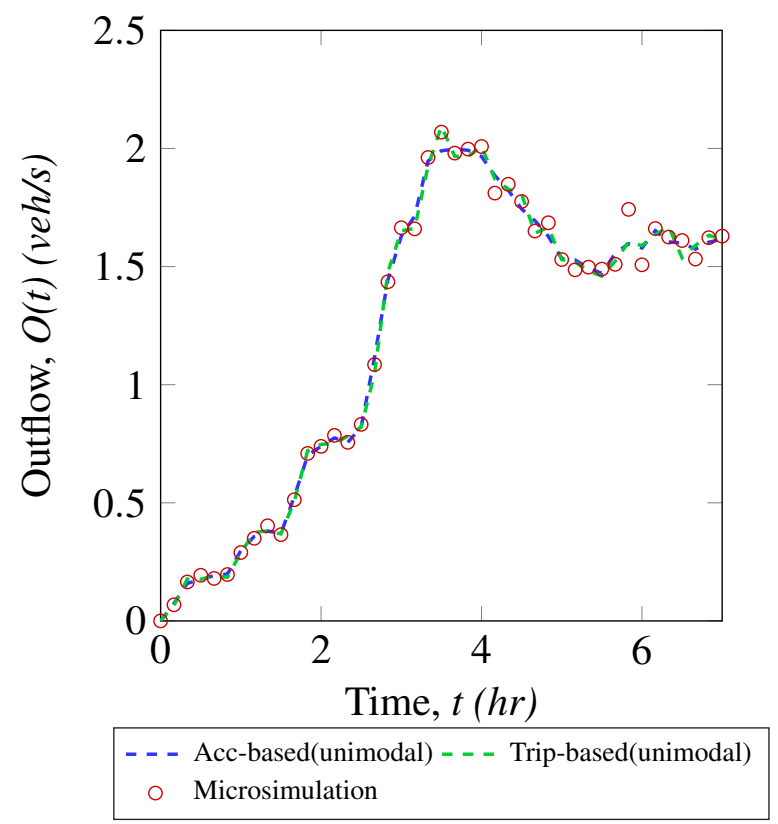

(c) Evolution of outflow with time.

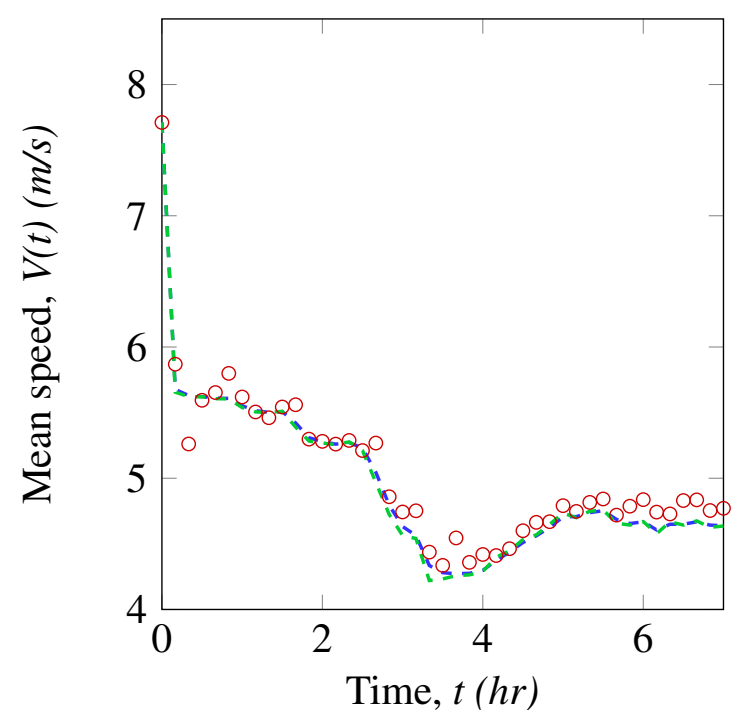

- - - Acc-based(unimodal) - - - Trip-based(unimodal) - Microsimulation

(b) Evolution of mean speed with time.

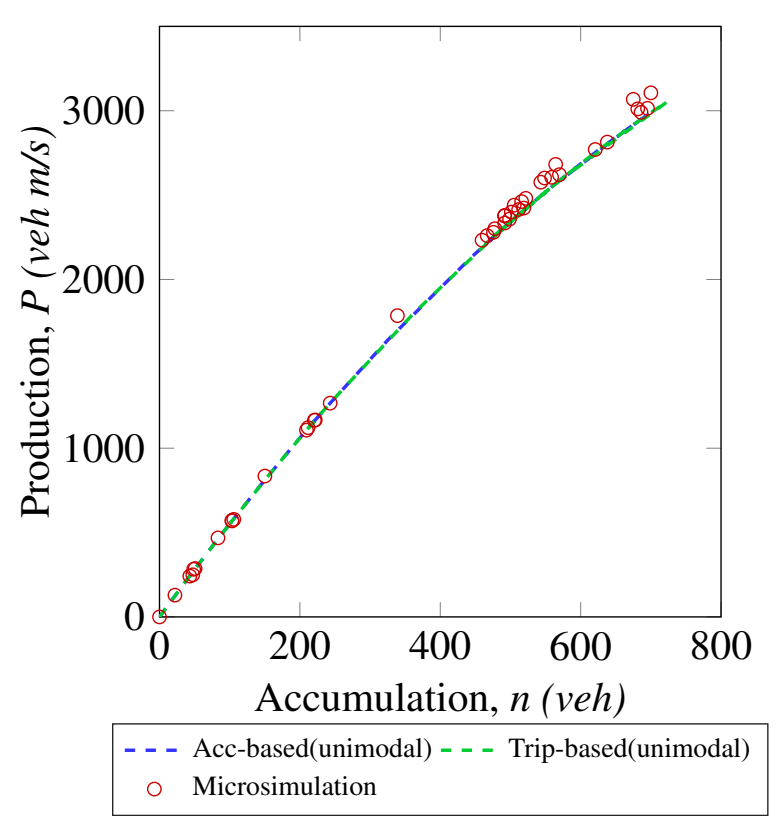

(d) Production MFD.

FIGURE 4 : Results of MFD-based approaches and micro-simulations corresponding to free flow demand scenario. OD trips estimation method is used in MFD-based models. 
pattern. Since the case of individual trip lengths is not considered for accumulation-based model, for the sake of comparison, four different trip lengths based on origin and destination is considered to present results for both accumulation-based and trip-based models. It should be noted that all the variables from MFD simulations are aggregated for $600 \mathrm{sec}$ in order to be able to compare with micro-simulations. It can observed that both approaches of MFD simulators provide a good approximation compared to micro-simulation. The absence of significant hysteresis in production is evident from Fig. 4d. The absence of hysteresis is in-line with the conclusions of the previous work Leclercq and Paipuri (18) and it is due to the smaller drop in the demand profile. The $\mathscr{L}_{2}$ norms of the error in accumulation compared to micro-simulation for trip-based and accumulationbased are 0.0241 and 0.0273 , respectively. The outflow in this case is defined as the trip completion rate of all the vehicles that travel in Lyon 6. The outflow of the micro-simulation is computed based on the trip ending times of each vehicle inside in Lyon 6. This method of computation of outflow includes all trips irrespective of origins and destinations. Hence, trip completion rate can be estimated accurately from micro-simulations. As observed in the case of accumulation, outflow is also well captured by the MFD simulations as shown in Fig. 4c. Hence, it can be concluded that both accumulation-based and trip-based models are verified in the free-flow regime using microsimulation data. The results of the present free-flow scenario with different trip length estimation methods have not exhibited any significant differences. Therefore, accuracies of MFD models with respect to $\mathrm{p}$-MFD fits and trip length estimation methods are discussed in the following section with a peak demand close to network saturation.

\section{Network saturation traffic state scenario}

In this section, a demand profile is considered such that the network is loaded close to the saturation. Figure 2a shows the normalized demand (in red) given to each OD pair in the microsimulation. As explained earlier, demand for MFD simulators is computed from the inflow cumulative curve of micro-simulation in Lyon 6 area. Figure 5a shows the accumulation evolution with time for accumulation-based, trip-based with both unimodal and bimodal p-MFD fits and microsimulation data. It can be noticed that the peak accumulation exceeds the critical value $\left(n_{c}=900\right)$ and network is saturated. The trip-based unimodal p-MFD approach over-predicts the peak accumulation by 91 veh as presented in Table 1 . This is due to use of mean p-MFD fit, which results in lower mean speeds and higher accumulation. Note that the accuracy of the unimodal trip-based approach improves both in $\mathscr{L}_{2}$ and $\mathscr{L}_{\infty}$ norms as the description of trip lengths is refined. On the other hand, accumulation-based model with unimodal p-MFD fit yields results that are closer to micro-simulation ones than the bimodal case. Using the unimodal p-MFD, production is estimated incorrectly in accumulated-based model, however outflow is well predicted, see Figs. 5d and 5c. Since, the key element of the accumulation-based model is outflow, accumulation evolution is well captured as shown in Fig. 5a. The comparison of $\mathscr{L}_{2}$ norms of accumulation-based and trip-based models with unimodal fit for corresponding trip length estimation infers that the models are very close in terms of accuracy. Besides, the evolution of mean speed and outflow presented in Figs. 5b and $5 \mathrm{c}$, respectively, are very similar for both MFD models with unimodal approach. As shown in Fig. 5d the hysteresis phenomenon cannot be reproduced using unimodal p-MFD fits for both MFD models. In the case of accumulation-based approach with unimodal fit, errors increase as the trip length description is refined. However, errors in outflow are very similar for all trip length estimation methods, which suggests that there is significant error in production compared to trip lengths. The $\mathscr{L}_{2}$ norms of mean speed and outflow for both accumulation-based and trip-based 


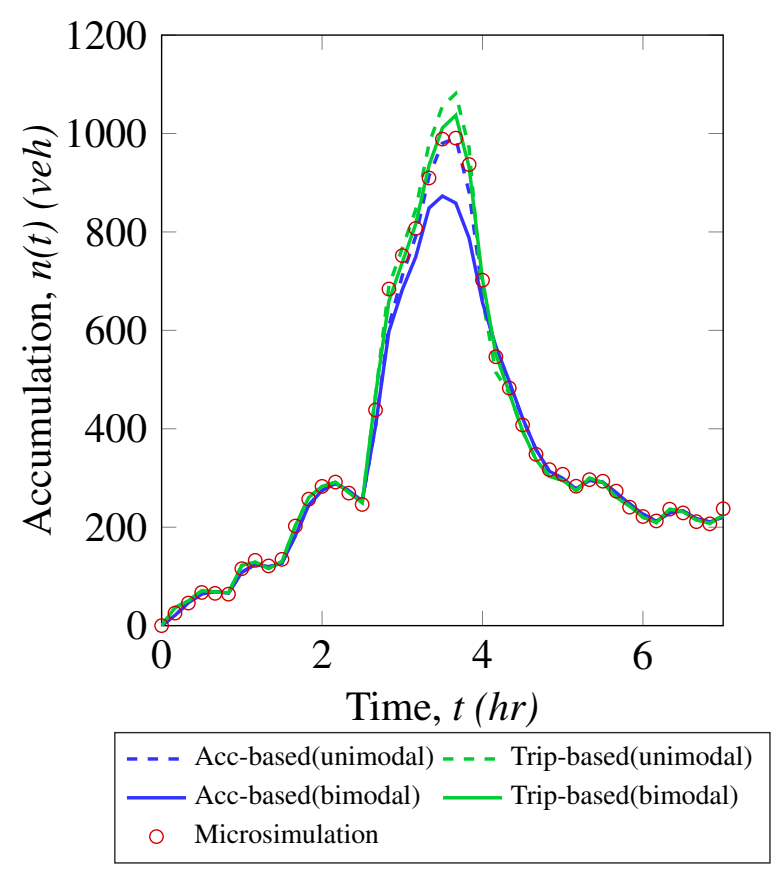

(a) Evolution of accumulation with time.

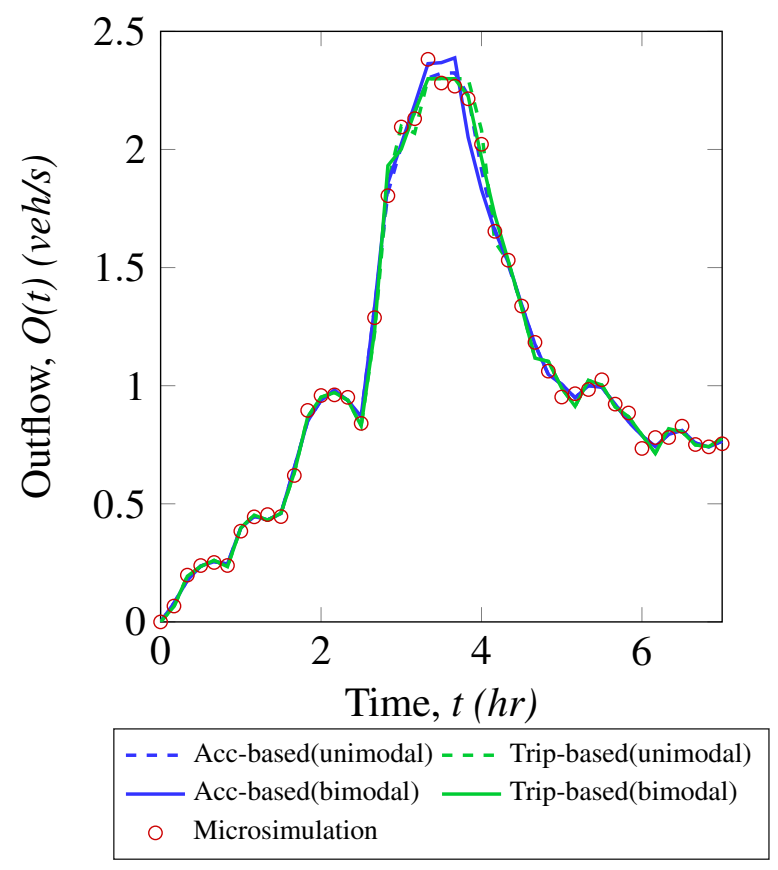

(c) Evolution of outflow with time.

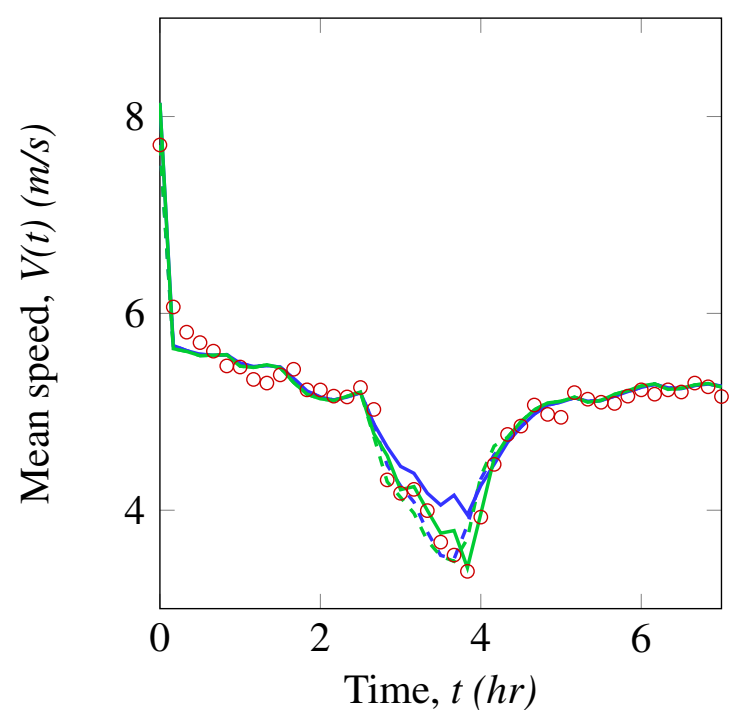

- - - Acc-based(unimodal) - - - Trip-based(unimodal) — Acc-based(bimodal) — Trip-based(bimodal) - Microsimulation

(b) Evolution of mean speed with time.

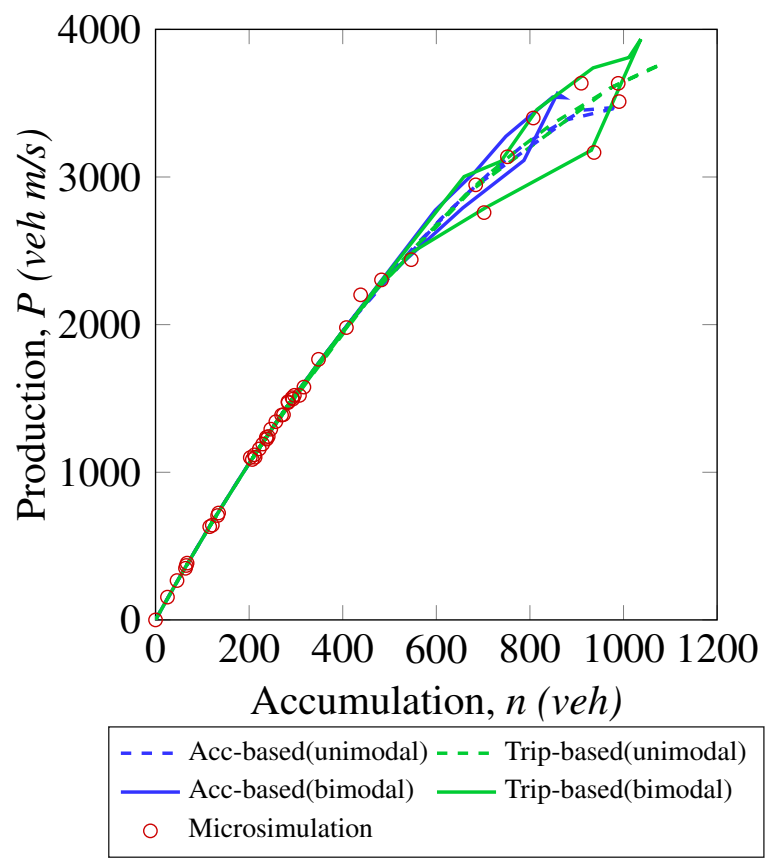

(d) Production MFD.

FIGURE 5 : Results of MFD-based approaches and micro-simulations corresponding to saturation flow demand scenario. OD trips estimation method is used in MFD-based models. 


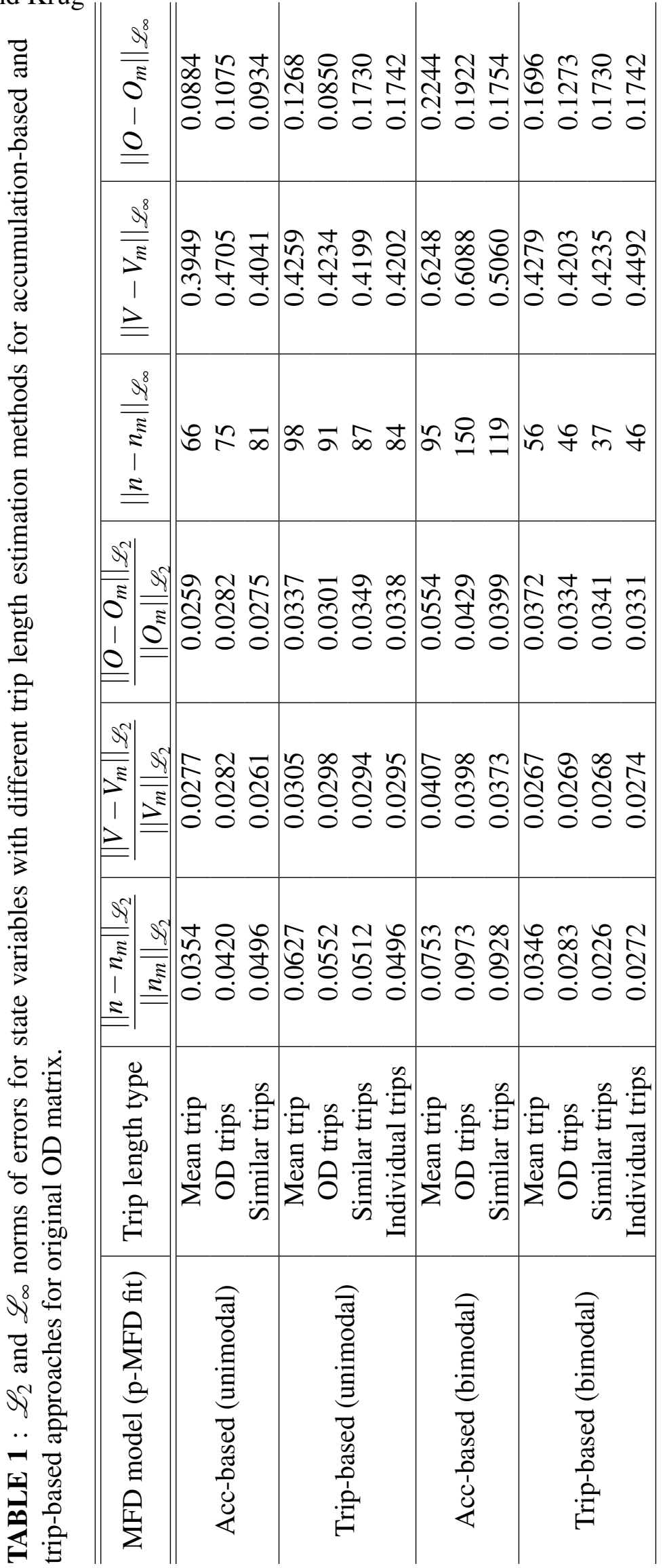


with unimodal p-MFD fits are comparable for all trip length estimation methods without a specific trend. As stated already, this can be due to larger error in p-MFD estimation.

Now comparing the bimodal accumulation-based and trip-based approaches, from Fig. 5a, it can be observed that trip-based approach with bimodal fit is more closer to micro-simulation than its counterpart. The most accurate solution is obtained using the clustered trip lengths estimation method using trip-based approach with bimodal p-MFD fit. Using a single trip with mean trip length gives the least accurate estimation of accumulation in the case of trip-based approach with bimodal fit. Figure $2 \mathrm{c}$ presented earlier shows that the trip lengths vary from $1 \mathrm{~m}$ to $4500 \mathrm{~m}$. By taking a single trip with mean trip length, vehicles may travel longer distances, which results in higher accumulation. This can be improved by taking a weighted mean of all trip lengths based on demand per each trip. However, this data will not be readily available in the practical applications. This conclusion complies with the formulation of trip-based approach when the trip lengths are distributed over wide range. Just like in the case of unimodal p-MFD, the accuracy of trip-based solutions with bimodal fit improves as trip length description is refined except for the case of individual trip lengths. The $\mathscr{L}_{2}$ and $\mathscr{L}_{\infty}$ error norms of trip-based model with bimodal fit with individual trip length case are larger than clustered trips. This might be due to errors from meanspeed approximation is the dominant compared to trip-length distribution in the case of individual trip lengths. A similar trend is observed in both outflow and mean speed for the case of tripbased approach with bimodal fit. On the other hand, error in accumulation in the case of bimodal accumulation-based approach increases as the trip-lengths are refined. It can be concluded that in the case of bimodal accumulation-based approach, considering a single trip with mean trip length produces satisfactory results. However, it is worth noting that the errors of outflow decreases with increasing the refinement in trip lengths. It suggests that production is well estimated and dominant errors in outflow are due to the approximation of trip lengths. It infers that refining the trip lengths results in better estimation of outflow. The reason for the opposite trend in accumulation might be due to the presence of numerical dissipation in inflow computations. The $\mathscr{L}_{2}$ norm of error for inflow cumulative curve between accumulation-based model and micro-simulation for mean trip case is $6.1 \times 10^{-4}$, which is two orders lower than errors obtained in accumulation. However, as the number of trip lengths increase with fewer trips on each trip length in the reservoir, the error in inflow cumulative curve can influence the error in accumulation evolution. Finally, Fig. 5d shows that the clock-wise hysteresis pattern is obtained by both accumulation-based and trip-based models. The size of the hysteresis loop in accumulation-based is smaller than the micro-simulation one because of the under-prediction of peak accumulation.

Overall, comparison of different models infers that trip-based with bimodal fit, similar trips and accumulation-based with unimodal p-MFD fit, mean trip models are very close to the microsimulation results and gives a good estimation of accumulation evolution for the real network of Lyon 6.

\section{Validation when changing the OD matrix}

The next part of the study is to compare the MFD models to the micro-simulation when OD matrix is modified, which changes the internal trip patterns. The estimated trip lengths from microsimulation is now shown in Fig. 2d. As stated earlier, the micro-simulations are carried out using static assignment by predefining the routes and their corresponding assignment coefficients. The OD matrix of Lyon 6 is changed artificially by increasing the flow between OD pairs which have longer trip lengths $(2000 m-3000 m)$ and decreasing the same amount of flow between OD pairs 
which have smaller trip lengths $(1000 m-2000 m)$. The idea is not to obtain a realistic scenario, but to create enough modifications in the trip patterns to have a significant differences from the reference scenarios. The accumulation-based MFD is applied with a unimodal p-MFD fit, while the trip-based is applied with a bimodal one.

\section{Without re-calibration of $p-M F D$ fit and trip lengths}

In this part, the results are presented using the same p-MFD fits and trip lengths proposed for the original OD matrix. Such a situation arises when the modeler does not consider the OD matrix changes and uses the previous calibration settings. The normalized demand pattern is same as the network saturation case as shown in Fig. 2a, however the actual demand is slightly different from the case of original OD matrix as shown in Fig. 2b. The results of accumulation-based with unimodal p-MFD fit, mean trip and trip-based with bimodal fit, OD trips are presented. Figure 6 presents the results of MFD models and micro-simulation. It can be observed that evolution of accumulation and mean speed are inaccurate for MFD models, especially during the network recovery phase. The hysteresis loop obtained in the p-MFD from micro-simulation is comparatively bigger than MFD models as shown in Fig. 6d. Figure 6c shows that both accumulation-based and trip-based models estimate the outflow evolution with a good accuracy. Since, the inflow for both models are equal, albeit the numerical errors, the difference in the accumulation is due to the inconsistencies in p-MFD fits and trip lengths.

\section{With re-calibration of only trip lengths}

This part shows the results with re-calibration of trip lengths according to the modified OD matrix, however using the same p-MFD fit for the original scenario. Figure 7 presents the results for different state variables for both MFD and micro-simulation models. The first noticeable different between previous results in Fig. 6 and the present one is that the peak accumulation in the accumulation-based model is over-predicted by about $50 \%$. The reason is that the mean trip length in the modified OD matrix case is $1652 \mathrm{~m}$ compared to $1505 \mathrm{~m}$ in the original OD matrix scenario. Hence, by using the same p-MFD fit as the original OD matrix case results in the smaller outflow and higher accumulation. The reduction of outflow can be observed in Fig. 7c for the accumulation-based model. On the other hand, the accuracy of the trip-based model is improved close to the peak accumulation using the re-calibrated trip lengths. However, the production hysteresis in the trip-based model is still not close to micro-simulation.

\section{With re-calibrated $p$-MFD fit and trip lengths}

Figure 8 shows the evolution of accumulation, mean speed, outflow with time along with p-MFD obtained from MFD models and micro-simulation using re-calibrated trip lengths and p-MFD fits. It can be noticed that the hysteresis loop in the p-MFD is improved for the trip-based model compared to Figs. $6 \mathrm{~d}$ and $7 \mathrm{~d}$. The p-MFD during the recovery phase of network in trip-based model is following the micro-simulation results. The consequence of this can be noticed in the evolution of accumulation, where accumulation values are higher during the recovery phase in Fig 8a compared to Fig. 7a. This highlights the importance of re-calibrating the p-MFD fit to accurately predict the transient state, especially during the network recovery. It can also be noticed that the evolution of accumulation during loading phase with both re-calibrated p-MFD fit and original p-MFD fit is very close. Even though, the peak accumulation in both accumulation-based model with unimodal fit and bimodal trip-based models are very close, it can be observed that the accumulation-based 

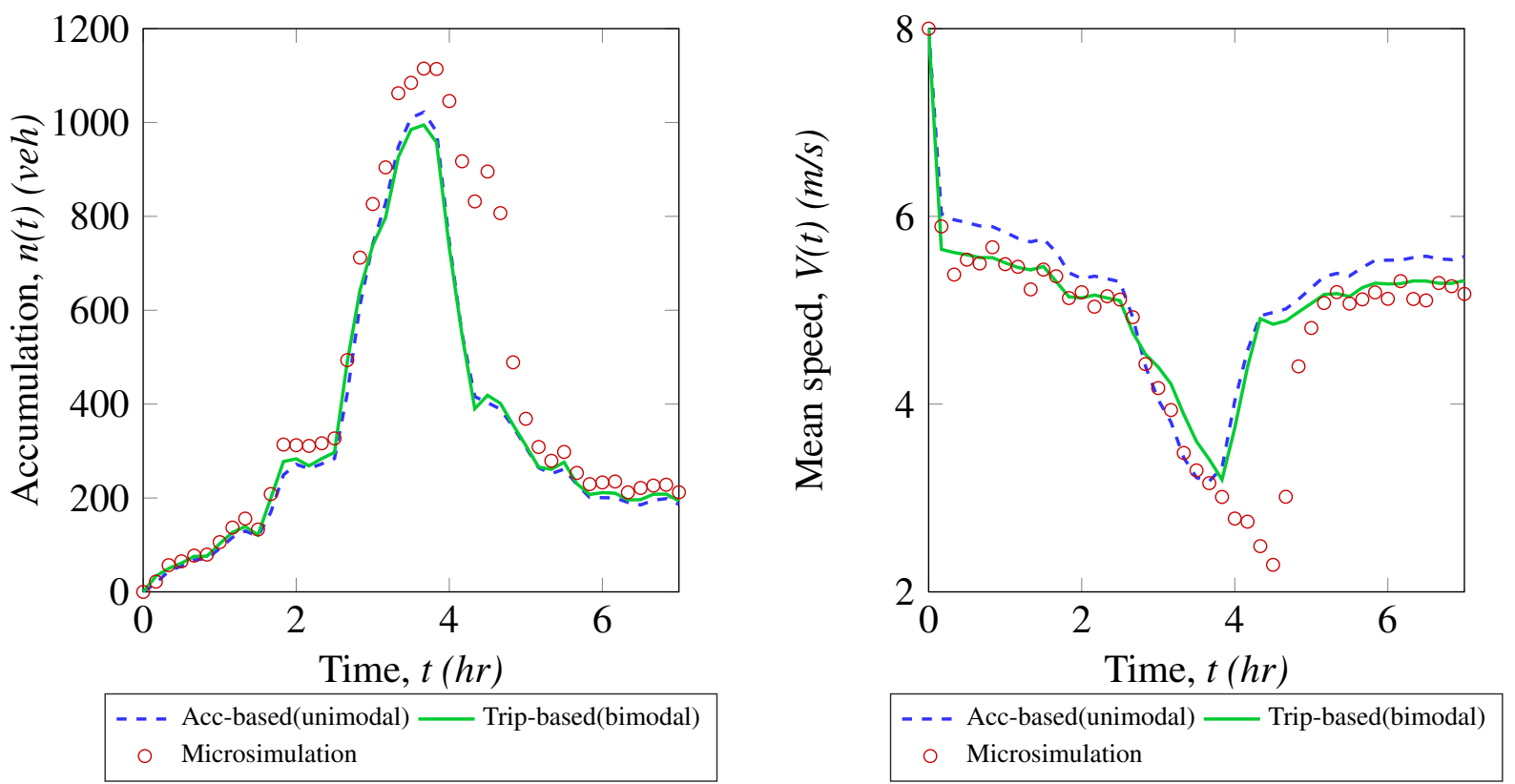

(a) Evolution of accumulation with time. Relative $\mathscr{L}_{2}$ error(b) Evolution of mean speed with time. Relative $\mathscr{L}_{2}$ error norm of acc-based is 0.2701 and trip-based is 0.1955 . norm of acc-based is 0.0817 and trip-based is 0.0905

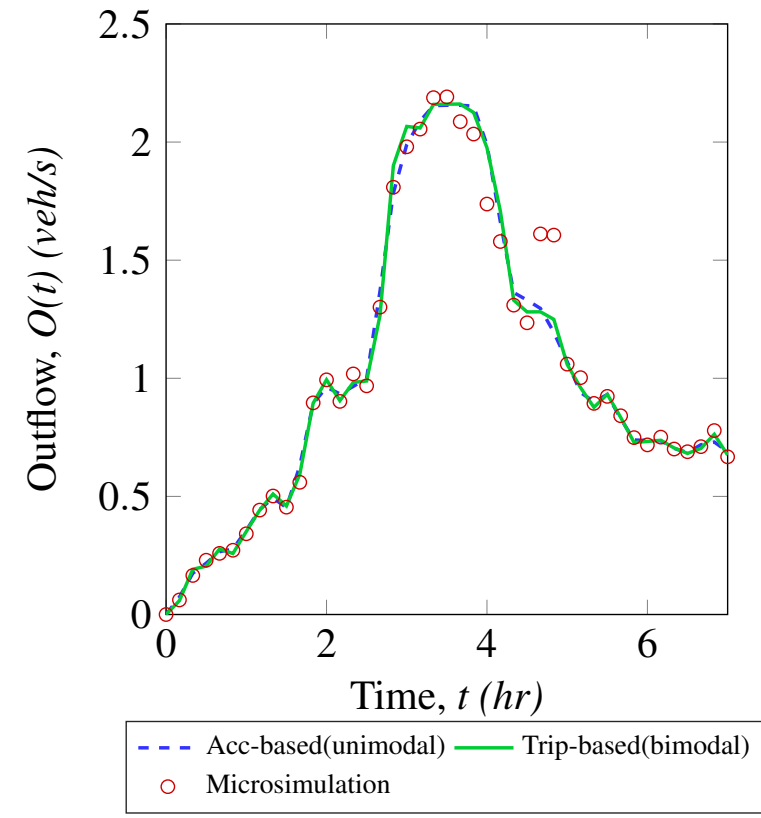

(c) Evolution of outflow with time. Relative $\mathscr{L}_{2}$ error norm of acc-based is 0.1600 and trip-based is 0.1071

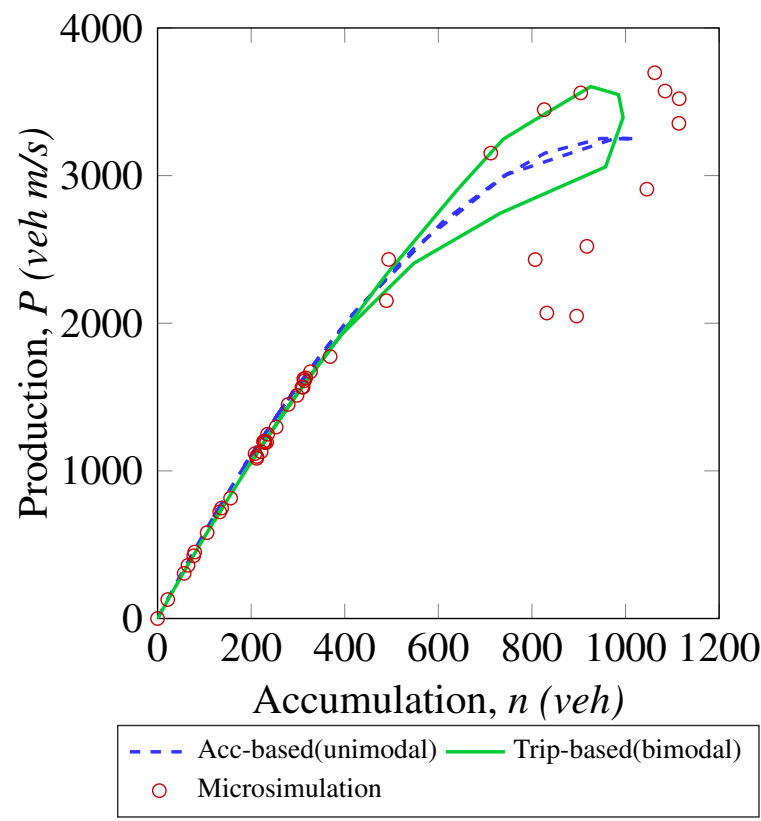

(d) Production MFD.

FIGURE 6 : Results of MFD-based approaches and micro-simulations corresponding to saturation flow demand scenario with modified OD matrix and without re-calibration of p-MFD fit and trip lengths. OD trips estimation method is used in MFD-based models. 

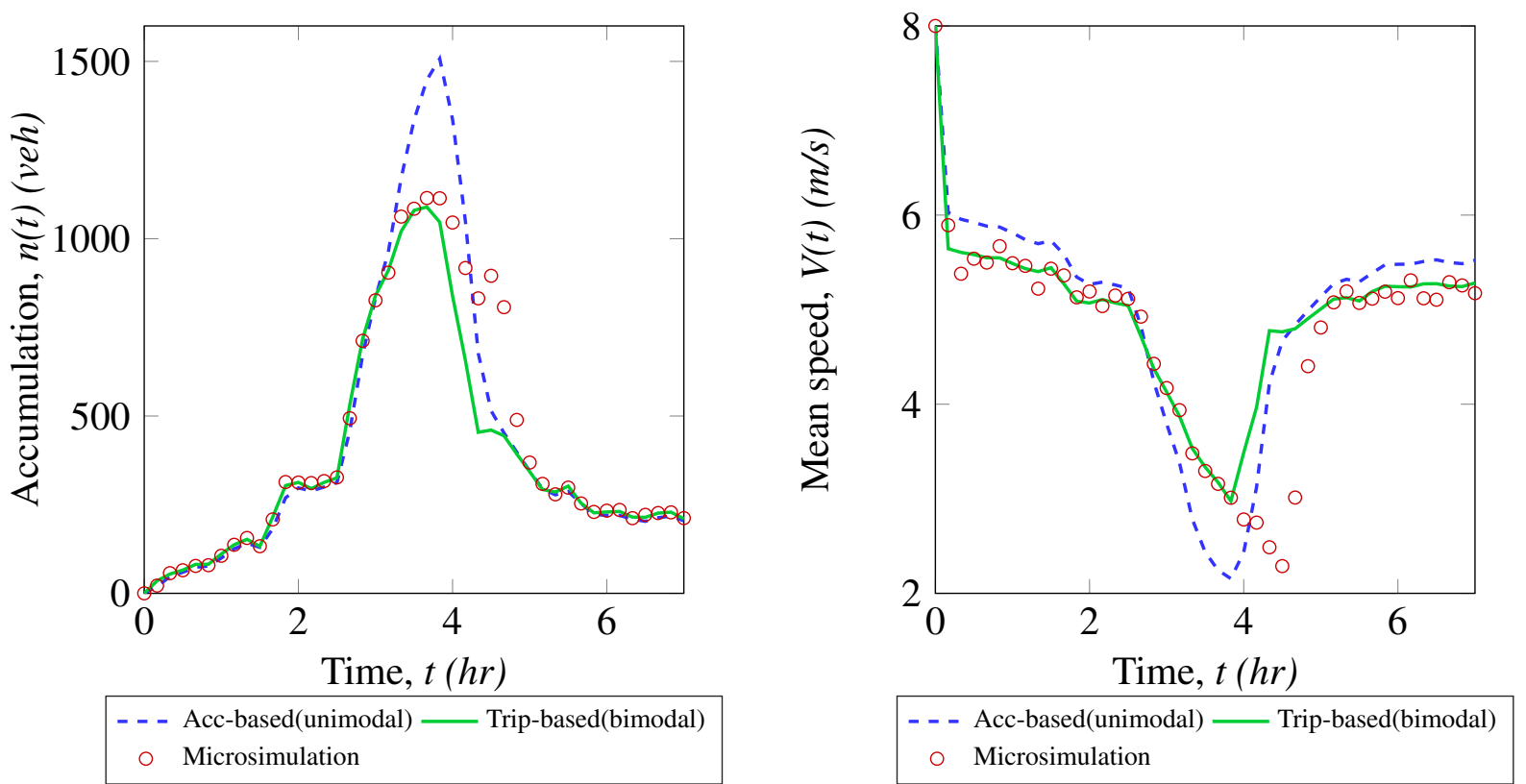

(a) Evolution of accumulation with time. Relative $\mathscr{L}_{2} \operatorname{error}(\mathrm{b})$ Evolution of mean speed with time. Relative $\mathscr{L}_{2}$ error norm of acc-based is 0.2427 and trip-based is 0.0793 norm of acc-based is 0.1344 and trip-based is 0.0365

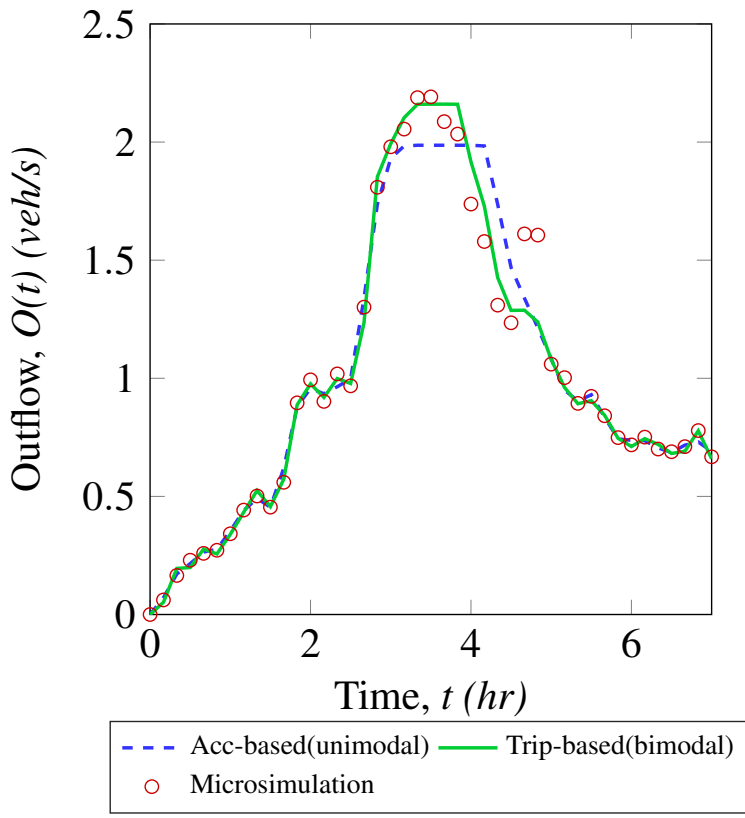

(c) Evolution of outflow with time. Relative $\mathscr{L}_{2}$ error norm of acc-based is 0.1170 and trip-based is 0.0543

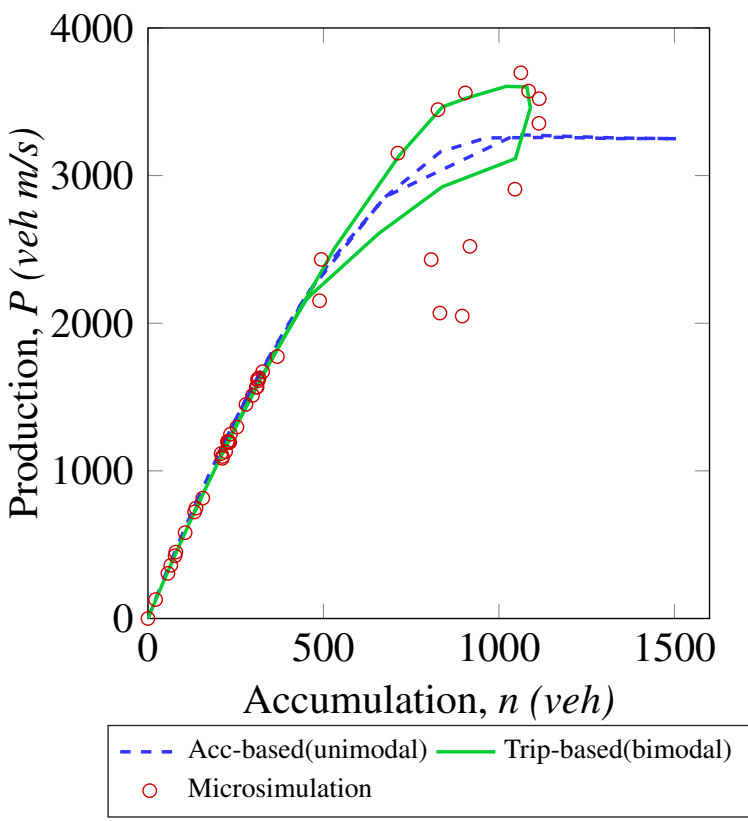

(d) Production MFD.

FIGURE 7 : Results of MFD-based approaches and micro-simulations corresponding to saturation flow demand scenario with modified OD matrix and with re-calibration of only trip lengths. OD trips estimation method is used in MFD-based models. 

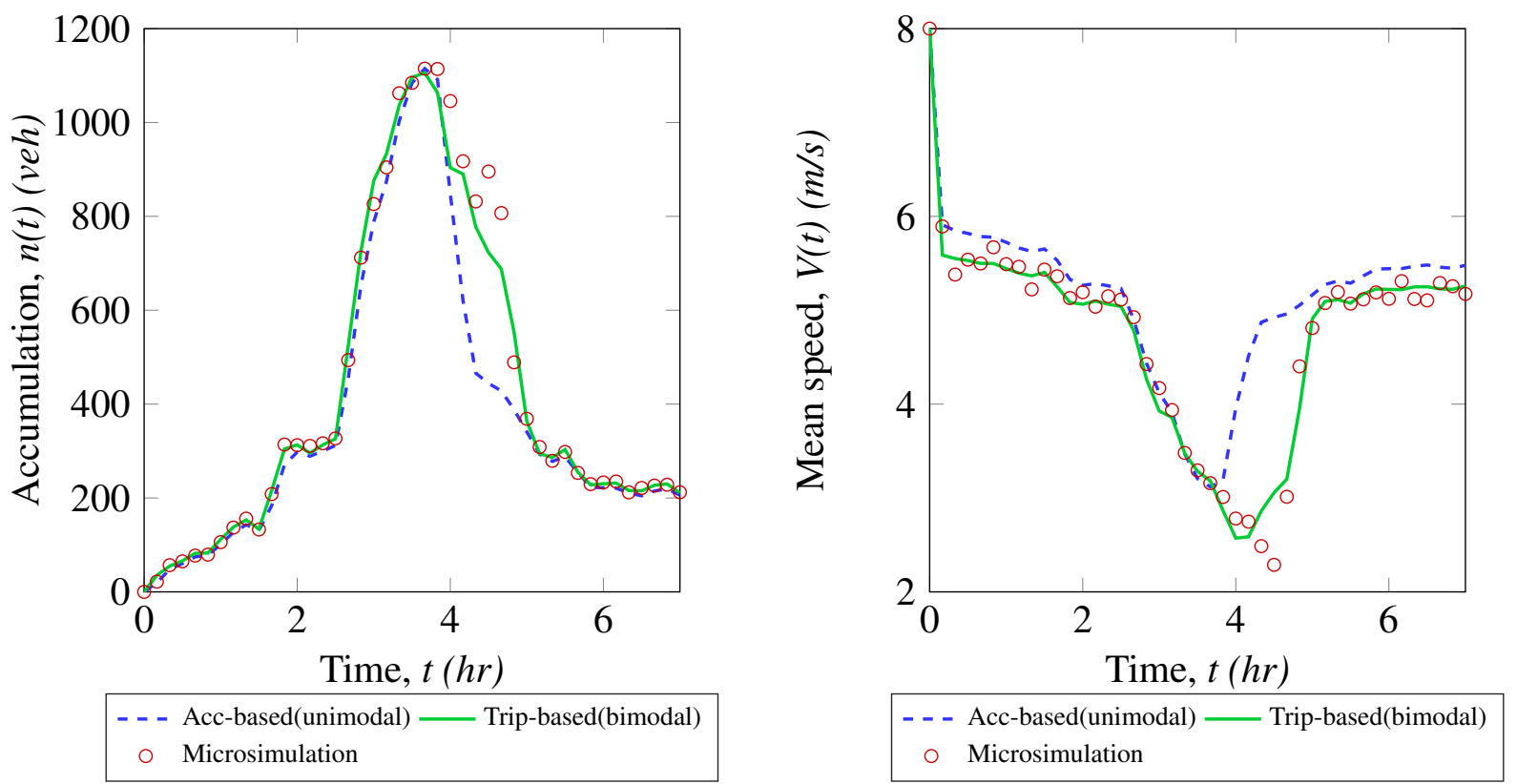

(a) Evolution of accumulation with time. Relative $\mathscr{L}_{2} \operatorname{error}(\mathrm{b})$ Evolution of mean speed with time. Relative $\mathscr{L}_{2}$ error norm of acc-based is 0.2223 and trip-based is 0.0638 norm of acc-based is 0.1510 and trip-based is 0.0383

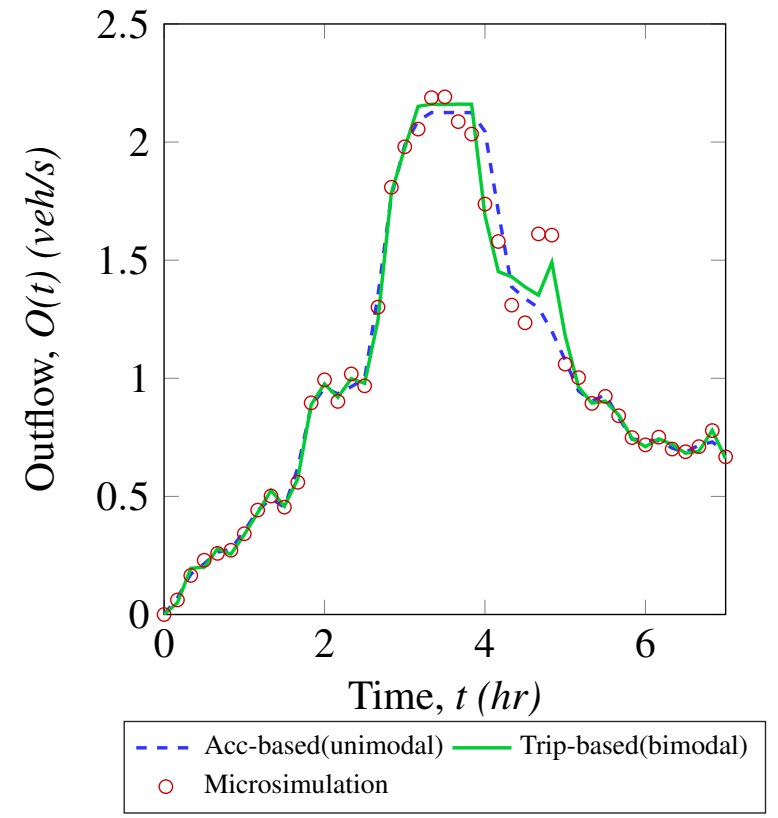

(c) Evolution of outflow with time. Relative $\mathscr{L}_{2}$ error norm of acc-based is 0.0850 and trip-based is 0.0561

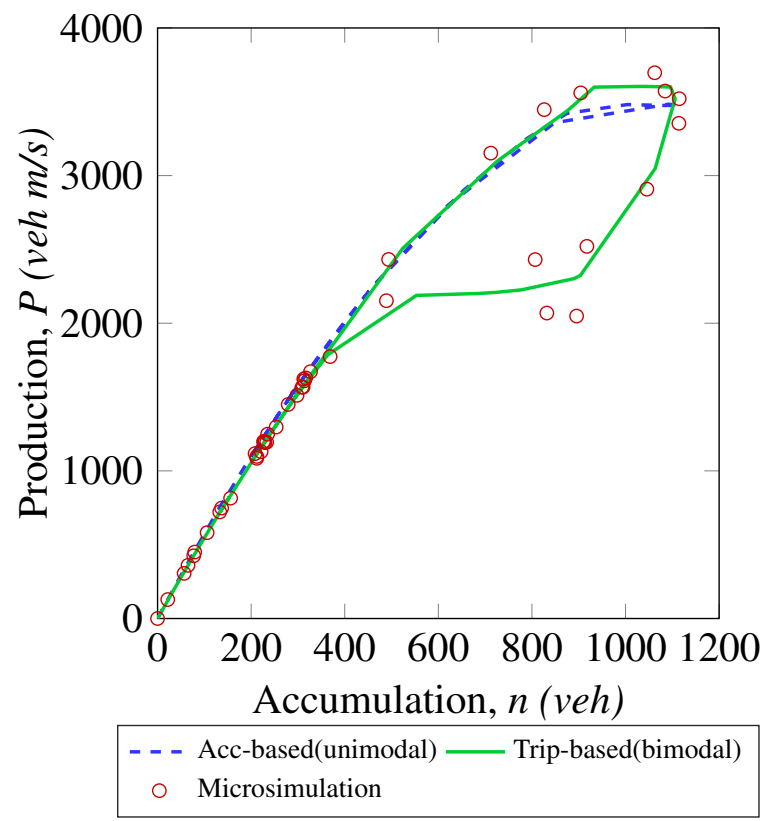

(d) Production MFD.

FIGURE 8 : Results of MFD-based approaches and micro-simulations corresponding to saturation flow demand scenario with modified OD matrix and with re-calibration of p-MFD fit and trip lengths. OD trips estimation method is used in MFD-based models. 
model's inability to estimate the production hysteresis accurately results in huge discrepancies of accumulation and mean speed in the network recovery phase as shown in Figs. 8a and 8b, respectively.

Hence, it can be concluded from this discussion that it is crucial to re-calibrate the pMFD fits and trip length distributions when the OD matrix is changed. The results infer that changes in OD matrix do not effect the network loading significantly, as noticed in Fig. 7d, where original p-MFD fit with calibrated trip lengths captured micro-simulation trend quite reasonably. However, re-calibration is necessary during the network recovery, as changes in OD matrix can have significant impact on the network unloading.

\section{CONCLUSIONS}

This work presents the calibration of p-MFD shape and trip length estimation of MFD-based approaches using validation of micro-simulation results on real network of Lyon 6. A reference free flow scenario and a network saturation scenario are presented to validate the MFD-based approaches. In addition, an additional case by changing the OD matrix is considered to study the impact of changes of OD matrix on accuracy of MFD simulations.

In the first case of free flow scenario, both accumulation-based and trip-based approaches gave satisfactory results using a unimodal p-MFD. Since, the network is largely in free flow regime and network unloading is slow, production hysteresis is negligible in this scenario. This test case is only used to benchmark the MFD-based approaches using micro-simulation data. In the following case of network saturation, clockwise hysteresis in the p-MFD is noticed from micro-simulations. The importance of considering a bimodal fit for p-MFD to capture the hysteresis pattern is demonstrated for the trip-based model. In the case of accumulation-based model, a good estimation of outflow and accumulation evolutions is obtained with unimodal p-MFD fit. The comparison of MFD-based approaches to micro-simulation results suggests that the MFD simulations can estimate the evolution of accumulation, mean speed and outflow to a good accuracy. It is concluded that the trip-based approach with bimodal p-MFD gives good estimates of state variables, however a more refined description of trip lengths results in more accurate results. Finally, the influence of changing OD matrix on the MFD simulations is studied. It is concluded that the re-calibration of p-MFD fit and trip lengths are required to accurately predict the dynamics of the network. 


\section{ACKNOWLEDGMENT}

This project has received funding from the European Research Council (ERC) under the European Union's Horizon 2020 research and innovation program (grant agreement No 646592 - MAGnUM project). The authors thank the reviewers for their insightful comments, which enhanced the quality of the paper.

\section{CONTRIBUTION STATEMENT}

The authors confirm contribution to the paper as follows: study conception and design: M. Paipuri, L. Leclercq; micro-simulation design and settings: M. Paipuri, J. Krug; analysis and interpretation of results: M. Paipuri, L. Leclercq; draft manuscript preparation: M. Paipuri. All authors reviewed the results and approved the final version of the manuscript.

\section{References} No. 7, 1969, pp. 323-327. pp. $495-515$. 21 for the year 2015. No. 9, 2008, pp. $771-781$.

[1] Godfrey, J., The mechanism of a road network. Traffic Engineering and Control, Vol. 11,

[2] Daganzo, C. F., Urban gridlock: Macroscopic modeling and mitigation approaches. Transportation Research Part B: Methodological, Vol. 41, No. 1, 2007, pp. 49 - 62.

[3] Geroliminis, N. and C. F. Daganzo, Existence of urban-scale macroscopic fundamental diagrams: Some experimental findings. Transportation Research Part B: Methodological, Vol. 42, No. 9, 2008, pp. 759 - 770.

[4] Knoop, V. L. and S. P. Hoogendoorn, Network transmission model: a dynamic traffic model at network level. In Transportation Research Board 93rd Annual Meeting Transportation Research Board, Washington, 2014, 14-1104.

[5] Yildirimoglu, M. and N. Geroliminis, Approximating dynamic equilibrium conditions with macroscopic fundamental diagrams. Transportation Research Part B: Methodological, Vol. 70, 2014, pp. $186-200$.

[6] Keyvan-Ekbatani, M., A. Kouvelas, I. Papamichail, and M. Papageorgiou, Exploiting the fundamental diagram of urban networks for feedback-based gating. Transportation Research Part B: Methodological, Vol. 46, No. 10, 2012, pp. 1393 - 1403.

[7] Haddad, J. and B. Mirkin, Coordinated distributed adaptive perimeter control for large-scale urban road networks. Transportation Research Part C: Emerging Technologies, Vol. 77, 2017,

[8] Ampountolas, K., N. Zheng, and N. Geroliminis, Macroscopic modelling and robust control of bi-modal multi-region urban road networks. Transportation Research Part B: Methodological, Vol. 104, 2017, pp. 616 - 637.

[9] Cao, J. and M. Menendez, System dynamics of urban traffic based on its parking-relatedstates. Transportation Research Part B: Methodological, Vol. 81, 2015, pp. 718 - 736, iSTTT

[10] Leclercq, L., A. Sénécat, and G. Mariotte, Dynamic macroscopic simulation of on-street parking search: A trip-based approach. Transportation Research Part B: Methodological, Vol. 101, 2017, pp. $268-282$.

[11] Daganzo, C. F. and N. Geroliminis, An analytical approximation for the macroscopic fundamental diagram of urban traffic. Transportation Research Part B: Methodological, Vol. 42,

[12] Buisson, C. and C. Ladier, Exploring the Impact of Homogeneity of Traffic Measurements 
1 on the Existence of Macroscopic Fundamental Diagrams. Transportation Research Record: Journal of the Transportation Research Board, Vol. 2124, 2009, pp. 127-136.

[13] Gayah, V. V. and C. F. Daganzo, Clockwise hysteresis loops in the Macroscopic Fundamental Diagram: An effect of network instability. Transportation Research Part B: Methodological, Vol. 45, No. 4, 2011, pp. 643 - 655.

[14] Geroliminis, N. and J. Sun, Hysteresis phenomena of a Macroscopic Fundamental Diagram in freeway networks. Transportation Research Part A: Policy and Practice, Vol. 45, No. 9, 2011, pp. 966 - 979, select Papers from the 19th International Symposium on Transportation and Traffic Theory (ISTTT).

[15] Ramezani, M., J. Haddad, and N. Geroliminis, Dynamics of heterogeneity in urban networks: aggregated traffic modeling and hierarchical control. Transportation Research Part B: Methodological, Vol. 74, 2015, pp. 1 - 19.

[16] Leclercq, L., C. Parzani, V. L. Knoop, J. Amourette, and S. P. Hoogendoorn, Macroscopic traffic dynamics with heterogeneous route patterns. Transportation Research Part C: Emerging Technologies, Vol. 59, 2015, pp. 292 - 307, special Issue on International Symposium on Transportation and Traffic Theory.

[17] Mahmassani, H. S., M. Saberi, and A. Zockaie, Urban network gridlock: Theory, characteristics, and dynamics. Transportation Research Part C: Emerging Technologies, Vol. 36, 2013, pp. $480-497$.

[18] Leclercq, L. and M. Paipuri, Macroscopic traffic dynamics in reservoirs under fast-varying demand profiles. Transportation Science, 2018, (Submitted).

[19] Kouvelas, A., M. Saeedmanesh, and N. Geroliminis, Enhancing model-based feedback perimeter control with data-driven online adaptive optimization. Transportation Research Part B: Methodological, Vol. 96, 2017, pp. $26-45$.

[20] Geroliminis, N., Dynamics of peak hour and effect of parking for congested cities. In Transportation Research Board 88th Annual Meeting Transportation Research Board, Washington, 2009, 09-1685.

[21] Yildirimoglu, M., M. Ramezani, and N. Geroliminis, Equilibrium analysis and route guidance in large-scale networks with MFD dynamics. Transportation Research Part C: Emerging Technologies, Vol. 59, 2015, pp. 404 - 420, special Issue on International Symposium on Transportation and Traffic Theory.

[22] Arnott, R., A bathtub model of downtown traffic congestion. Journal of Urban Economics, Vol. 76, 2013, pp. $110-121$.

[23] Daganzo, C. F. and L. J. Lehe, Distance-dependent congestion pricing for downtown zones. Transportation Research Part B: Methodological, Vol. 75, 2015, pp. 89 - 99.

[24] Lamotte, R. and N. Geroliminis, The Morning Commute in Urban Areas: Insights from Theory and Simulation. In Transportation Research Board 95th Annual Meeting Transportation Research Board, Washington, 2016, 16-2003.

[25] Mariotte, G., L. Leclercq, and J. A. Laval, Macroscopic urban dynamics: Analytical and numerical comparisons of existing models. Transportation Research Part B: Methodological, Vol. 101, 2017, pp. $245-267$.

[26] Mariotte, G. and L. Leclercq, Flow exchanges in multi-reservoir systems with spillbacks. Transportation Research Part B: Methodological, 2018, (Submitted).

[27] Newell, G., A simplified car-following theory: a lower order model. Transportation Research Part B: Methodological, Vol. 36, No. 3, 2002, pp. 195 - 205. 
[28] Leclercq, L., J. Laval, and E. Chevallier, The Lagrangian coordinates and what it means for first order traffic flow models. In Proceedings of the 17th International Symposium on Transportation and Traffic Theory, Elsevier, London, 2007, 735-753.

[29] Dial, R. B., A probabilistic multipath traffic assignment model which obviates path enumeration. Transportation Research, Vol. 5, No. 2, 1971, pp. 83 - 111.

[30] Mariotte, G. and L. Leclercq, MFD-Based Simulation: Spillbacks in Multi-Reservoir Networks. In Transportation Research Board 97th Annual Meeting Transportation Research Board, Washington, 2018, 18-04679.

[31] Lamotte, R. and N. Geroliminis, The morning commute in urban areas with heterogeneous trip lengths. Transportation Research Part B: Methodological, 2017.

[32] Merchant, D. K. and G. L. Nemhauser, A Model and an Algorithm for the Dynamic Traffic Assignment Problems. Transportation Science, Vol. 12, No. 3, 1978, pp. 183-199.

[33] Friesz, T. L., J. Luque, R. L. Tobin, and B.-W. Wie, Dynamic Network Traffic Assignment Considered as a Continuous Time Optimal Control Problem. Operations Research, Vol. 37, No. 6, 1989, pp. 893-901. 